\title{
Tactile Semiotics: The Meanings of Touch Explored with Low-tech Prototypes
}

\author{
Simone Gumtau* \\ (*)Simone Gumtau University of Portsmouth, UK \\ E-mail: simone.gumtau@port.ac.uk
}

\begin{abstract}
In this paper I will describe the term Tactile Semiotics - the meanings we ascribe to different types of haptic sensations. When designing devices for haptic communication or haptic input devices for HCI, it makes sense firstly to look to psychophysics as an informant. However, to gain insight into tactility as both experience and meaning, it may also be beneficial to look at the cultural and social context of touch.

Also, I will explain how it is possible to explore this field with the help of low-tech prototypes and methodologies more common in art and design.
\end{abstract}

\section{Introduction}

\subsection{Touch as Communication Medium}

It has been established that human beings can learn new languages based on touch. Tadoma and Braille as communication systems for people with sensory impairments are obvious examples of this. Projects like ComTouch [1] and Haptic Icons [2] are going a step further by attempting to convey meaning within different forms of haptic elements and to utilize our intuitive, immediate sense of touch. With creating the Vibrobod and What's Shaking, researchers Dobson et al [3] found vibration and temperature successfully facilitated the exchange of emotional and social content. They conclude their mappings were so successful that no prior training was needed to use and understand their devices. Dobson et al have tapped into an area of research potential which offers great benefits for extending the communicative capacity of haptic interfaces.

\subsection{Semiotics to the Rescue}

What is needed and called for by most researchers of haptic communication devices is a set of general rules in haptic communication that could inform a system of design parameters. Too little is known about the exact communication possibilities of touch - although many have a pretty good "feeling" about it. There may be an underlying, learned system of signs and symbols, a cultural and social system of codes we can build on: semiotics. This may be what has made projects like the Vibrobod and What's Shaking successful - they have utilized this system to map meaning to touch. Semiotics is the science of signs. Every language is a system of signs, and therefore, to study the generation of meaning and communication effectively, it is important to be aware of semiotics. In Graphic Design - visual communication - the study of semiotics and visual language is one of the foundations to effectively express and communicate a message. According to semiotics theory, a coding system is existent in every media channel [4], although researchers have concentrated mostly on linguistics and aesthetics. If we can familiarize ourselves with the codes of tactile semiotics, can we utilize them in haptic communication?

\section{Tactile Semiotics / Haptic Expression}

\subsection{Association with Textures: Haptic Box}

Haptic Box is designed to investigate whether we associate certain emotional values with tactile experiences. It is a box containing ten different textures mounted on cardboard, presented in a random sequence. They can be felt (not seen) with one hand while the other hand fills out a semantic differential scale. This is a set of 12 polarized word pairs rated from one to seven. It is designed to show the semantic link between the tested object and the subject's mental imagery. A questionnaire about touch memories and associations was filled out after the touching to gain insight in to the subject's touch awareness.

2.1.1 Association to Physical Nature. A lot of overlap on the collated scales was probably often due to the physical nature of the object, and the fact that some of the word pairs can be taken very literally e.g., almost everyone associated rugged rather than 
delicate with the Tree Fungus. Whereas no one could agree whether the Fungus was pleasurable or unpleasurable to touch.

2.1.2 Cross-modal Association. The Silk texture most found it to be delicate and light (physical feature), but also almost all subjects associated it to be fragrant rather than foul. It is possible that we are entering the world of cross-modal associations here: to associate Silk with a feminine element, maybe with perfume. People also agreed on it feeling sweet, young, relaxed, valuable, but interestingly, weak and cowardly: terms traditionally associated with femininity - the "weak" gender.

\subsubsection{Common Association with Natural Materials.} Noticeably, it seems that the most correlation between subjects is over the materials that are the most organic, natural. It appears the more artificial the material, the more the associations diverge. This could be because the settings in which we meet organic materials (Tree Fungus, Bark) are usually similar, so we share the same experiences and associations. The experience of synthetic materials on the other hand is manifold and it is more difficult to describe a common denominating experience or association.

11 subjects have undertaken the Haptic Box. There seems to be indication of a certain correlation between test subjects' choices - meaning there could be a cultural system of semiotics at work. This will be quantified in the next phase, a larger user study with slightly improved and quicker test procedures.

\subsection{Shapes may Communicate: PinKom}

With the PinKom - a mock-up prototype of an imaginary haptic device - I hope to study the potential of haptic communication. The functional device would be a tele-taction sensor/actuator system. Haptic expressions are envisaged in the form of a shape display driven by a pin mechanism. This could later be enhanced with vibration, temperature and textures. Touch is assumed to be very useful in communicating presence and affect to loved ones in remote locations or increase a sense of presence while interacting with digital environments. It also has a rich potential as a broadcasting medium [7].

The PinKom will be used by couples in a love relationship for at least a week. Qualitative data will be generated by letting intimate friends or lovers use the prototype over a prolonged time. Spontaneous forming of communication codes shall be monitored. We will gain insights into the real uses people would put their new touch based communication devices to.
Even though the prototype is not fully functional at present, it will give us some results and pointers for future designs. For example, it seems that the dimension of the real time force will be more powerful than the shape display on its own.

\section{Conclusion}

\subsection{Broadened Range of Ideas}

Haptic Box, using semiotics and semantic differentials, shows how methodologies from different disciplines can bring new valuable insights. PinKom, using a mock-up prototype rather than a functional one, is an example of the benefits of low-tech prototypes for quick user evaluation. Tollmar [7] suggests using a holistic approach to designing new interfaces. Blue sky research may result in failure due to lack of realistic outcomes. On the other hand, contemplating only the feasible may result in inhibition of innovative ideas.

With technology and toolkits becoming more accessible, there is a good chance that ideas will flow freely across research disciplines and designs will be varied - just as user's needs and preferences. This mix of approaches and ideas will help to build evocative interfaces using metaphor, semiotics and suggestion, resulting in an active, participative and imaginative interactive process - which so far has been most successful in creating a meaningful experience for the user.

\section{References}

[1] A. Chang, S. O'Modhrain, R. Jacob, E. Gunther, H. Ishii, "ComTouch: Design of a Vibrotactile Communication Device”, Proceedings of DIS '02, 2002 .

[2] K. E. MacLean, M. Enriquez, "Perceptual Design of Haptic Icons", Proceedings of EuroHaptics, 2003.

[3] K. Dobson, D. Boyd, W. Ju, J. Donath, H. Ishii, "Creating Visceral Personal and Social Interactions in Mediated Spaces”, Proceedings of CHI '01, 2001.

[4] W. Nöth, "Handbook of Semiotics", Bloomington, Indiana University Press, 1990.

[5] S. O’Modhrain, I. Oakley, "Adding Interactivity: Active Touch in Broadcast Media", proceedings of the $12^{\text {th }}$ Symposium on Haptic Interfaces for Virtual Environments and Teleoperator Systems, Chicago, 2004.

[6] K. Tollmar, S. Junestrand, O. Torgny, "Virtually Living Together - Using Multiple-Method Designing in the Search for Telematic Emotion", Proceedings of DIS ‘ $00,2000$. 